\title{
Material screening and choosing methods - A review
}

\begin{abstract}
The selection of a material for a specific engineering purpose is a lengthy and expensive process. Approximately always more than one material is suitable for an engineering application, and the final selection is a compromise that brings some advantages as well as disadvantages. One of the issues that emerges from this review is that regardless of the relation of design stages and process selection with material selection, screening and ranking are two vital steps in the material selection. A variety of quantitative selection procedures have been developed to solve this issue, so that a systematic evaluation can be made. This paper seeks to address the following questions: (1) what is the contribution of the literature in the field of screening and choosing the materials? (2) What are the methodologies/systems/tools for material selection of engineering components? (3) Which approaches were prevalently applied? (4) Is there any inadequacy of the approaches? This research not only provides evidence that the multi-criteria decision making approaches has the potential to greatly improve the material selection methodology, but also aids the researchers and decision makers in applying the approaches effectively.
\end{abstract}

Keyword: Material selection; Multi-criteria decision making; Selection for material properties $(\mathrm{H})$ 